\title{
Genetic variation of Plasmodium falciparum histidine-rich protein 2 and 3 in Assosa zone, Ethiopia: its impact on the performance of malaria rapid diagnostic tests
}

Gezahegn Solomon Alemayehu*, Alebachew Messele ${ }^{1}$, Kayla Blackburn² ${ }^{2}$ Karen Lopez ${ }^{2}$, Eugenia Lo ${ }^{3,4}$, Daniel Janies ${ }^{2}$ and Lemu Golassa' ${ }^{1}$

\begin{abstract}
Background: Rapid diagnostic tests (RDT) are commonly used for the diagnosis of malaria caused by Plasmodium falciparum. However, false negative results of RDT caused by genetic variation of $P$. falciparum histidine-rich protein 2 and 3 genes (pfhrp2/3) threaten existing malaria case management and control efforts. The main objective of this study was to investigate the genetic variations of the pfhrp2/3 genes.

Methods: A cross-sectional study was conducted from malaria symptomatic individuals in 2018 in Assosa zone, Ethiopia. Finger-prick blood samples were collected for RDT and microscopic examination of thick and thin blood films. Dried blood spots (DBS) were used for genomic parasite DNA extraction and molecular detection. Amplification of parasite DNA was made by quantitative PCR. DNA amplicons of pfhrp2/3 were purified and sequenced.

Results: The PfHRP2 amino acid repeat type isolates were less conserved compared to the PfHRP3 repeat type. Eleven and eight previously characterized PfHRP2 and PfHRP3 amino acid repeat types were identified, respectively. Type 1, 4 and 7 repeats were shared by PfHRP2 and PfHRP3 proteins. Type 2 repeats were found only in PfHRP2, while types 16 and 17 were found only in PFHRP3 with a high frequency in all isolates. 18 novel repeat types were found in PfHRP2 and 13 novel repeat types were found in PfHRP3 in single or multiple copies per isolate. The positivity rate for PfHRP2 RDT was high, 82.9\% in PfHRP2 and 84.3\% in PfHRP3 sequence isolates at parasitaemia levels $>250$ parasites/ $\mu \mathrm{l}$. Using the Baker model, $100 \%$ of the isolates in group A (If product of types $2 \times$ type 7 repeats $\geq 100$ ) and $73.7 \%$ of the isolates in group $B$ (If product of types $2 \times$ type 7 repeats 50-99) were predicted to be detected by PfHRP2 RDT at parasitaemia level $>250$ parasite/ $\mu$ l.
\end{abstract}

Conclusion: The findings of this study indicate the presence of different PfHRP2 and PfHRP3 amino acid repeat including novel repeats in P. falciparum from Ethiopia. These results indicate that there is a need to closely monitor the performance of PfHRP2 RDT associated with the genetic variation of the pfhrp2 and pfhrp3 gene in P. falciparum isolates at the country-wide level.

Keywords: RDT, Plasmodium falciparum, Genetic variation, PfHRP2, PfHRP3, Amino acid sequence, Repeat type, Assosa, Ethiopia

*Correspondence: gezasolo2020@gmail.com

${ }^{1}$ Addis Ababa University, Aklilu Lemma Institute of Pathobiology, Addis Ababa, Ethiopia

Full list of author information is available at the end of the article permits use, sharing, adaptation, distribution and reproduction in any medium or format, as long as you give appropriate credit to the original author(s) and the source, provide a link to the Creative Commons licence, and indicate if changes were made. The images or other third party material in this article are included in the article's Creative Commons licence, unless indicated otherwise in a credit line to the material. If material is not included in the article's Creative Commons licence and your intended use is not permitted by statutory regulation or exceeds the permitted use, you will need to obtain permission directly from the copyright holder. To view a copy of this licence, visit http://creativecommons.org/licenses/by/4.0/. The Creative Commons Public Domain Dedication waiver (http://creativeco mmons.org/publicdomain/zero/1.0/) applies to the data made available in this article, unless otherwise stated in a credit line to the data. 


\section{Background}

Malaria caused by Plasmodium falciparum has remained a life-threatening infectious disease despite significant advances made towards malaria control and elimination in most malaria endemic countries in the world [1]. Plasmodium falciparum is the most common species that causes complicated malaria in humans. The genetic variation in the genome of $P$. falciparum has the potential to make pathogenesis virulent and challenge the accuracy of diagnosis [2]. One of the strategic pillars to reduce the morbidity and mortality associated with falciparum malaria is the provision of rapid and accurate malaria diagnostic tools in malaria-endemic settings [3].

Rapid diagnostic tests (RDTs) are widely and routinely used in remote and resource limited settings. Currently available malaria RDTs utilize parasite-specific antigens produced by malaria parasites for diagnosis [4]. The most commonly used biomarkers in antigen-based malaria RDTs are lactate dehydrogenase (LDH), aldolase, and histidine-rich protein 2 (HRP2) [5, 6]. The PfHRP3 antigen, also produced by $P$. falciparum, has been shown to share high homology with PfHRP2 [7] and plays a substantial role in the detection of $P$. falciparum infections by PfHRP2-based RDTs [8].

The $p f h r p 2$ and $p f h r p 3$ genes are located in the subtelomeric region of chromosome 8 and 13, respectively. Both pfhrp 2 and $p f h r p 3$ genes consist of a single intron and two exons $[7,9]$. Exon 2 in both pfhrp 2 and pfhrp3 encodes amino acids, which exhibit high levels of homology, suggesting that these genes were originated by duplication and divergence from a common ancestral sequence [7, 10]. The amino acid sequences of PfHRP2 and PfHRP3 contain several tandem copies of alanine and histidinerich repeat. The PfHRP2/3 amino acid sequences with multiple repeats of $\mathrm{AHH}$ and AHHAAD contain approximately $35 \%$ histidine $(\mathrm{H}), 40 \%$ alanine $(\mathrm{A})$, and $12 \%$ aspartate (D) [7]. Baker et al. showed several epitopes in PfHRP2 and PfHRP3 such as type 2 (AHHAHHAAD), type $4(\mathrm{AHH})$, and type $7(\mathrm{AHH} A \mathrm{AD})$ that are targeted by monoclonal antibodies against PfHRP2 and PfHRP3 antigens based on the PfHRP2 RDTs [11, 12].

However, frequent recombinations that occur at the subtelomeric regions contribute to vast genetic variation in the Pfhrp 2 and pfhrp3 genes [8, 13, 14]. Recent studies from several African and South American countries showed that extensive variation in the amino acid sequences of PfHRP2 and PfHRP3 could influence the frequency of the respective epitopes recognized by monoclonal antibodies [8, 12, 15]. Furthermore, deletions of the $p f h r p 2 / 3$ gene cause a lack of target antigen in the diagnosis of $P$. falciparum malaria [16-18]. Genetic variation and deletion of PfHRP2 and PfHRP3 amino acid sequence cause false negative testing using
PfHRP2-based RDTs [19, 20]. Ultimately, inaccurate diagnosis threatens ongoing efforts in malaria control and prevention [21].

Before this study, there were no data on the genetic variation of the $p f h r p 2 / 3$ gene in Assosa zone, northwest Ethiopia. Information on the abundance of PfHRP2 and PfHRP3 peptides repeats in P. falciparum isolates from geographically distinct regions is vital to monitor the diagnostic performance of PfHRP2 RDTs. Thus, the aim of this study was to determine the extent of genetic variation of $p f h r p 2$ and $p f h r p 3$ in clinical isolates, where $P$. falciparum is highly prevalent and PfHRP2 RDT is used for front-line diagnosis.

\section{Methods \\ Study area and period}

This study was carried out during the low and high transmission seasons in four selected health facilities: Assosa, Bambasi, Kurmuk and Sherkole Health Centres in Assosa Zone, Benishangul-Gumuz regional state, northwest Ethiopia, April to December 2018. The study area is a high malaria transmission setting. The Assosa Zone is located on the border of Sudan, where transboundary transmission of malaria likely occurs. The map of the study area is indicated in Additional file 1.

\section{Study design, sample size and sampling technique}

A cross-sectional health facilities based study was conducted to assess genetic variation of $p f h r p 2 / 3$ in clinical isolates. The study populations were residents of the Assosa Zone which includes all patients with clinical suspicion of malaria aged $\geq 5$ years in the selected health facilities during the study period. The inclusion criteria were all study participants with clinical suspicion of malaria who gave their written consent and/or assent. High-quality sequence data were included for molecular analysis of $p f h r p 2 / 3$ genetic variation. Study subjects who worked or lived outside the Assosa Zone catchment area and or who were unwilling to participate were excluded from the study. Data with poor quality sequences were also excluded.

The sample size was calculated based on the single population proportion formula $n=z^{2} p(1-p) / d^{2}$ [22]; Where, $\mathrm{n}=$ the sample size, $\mathrm{z}=1.96$ at $95 \%$ confidence interval $(\mathrm{CI}), \mathrm{d}=$ margin of error at $5 \%, \mathrm{p}$ (expected malaria prevalence rate) is $40 \%$ prevalence of symptomatic malaria in a hospital study of the region [23]. As a result, the sample size calculated with $10 \%$ non-response was 406 study participants. A total of 812 study participants were involved in this study, 406 study participants in low and 406 study participants in high transmission seasons. 
The study area, Assosa Health Centre, Bambasi Health Centre, Kurmuk Health Centre and Sherkole Health Centre, was selected using a simple random sampling technique among eight districts in the Assosa zone. Then, allocation of the study participants to each selected health centre was performed based on proportion of confirmed malaria case in each selected district/Woreda.

\section{Sample collection}

In a single finger-prick, capillary blood samples were collected from malaria suspected individuals for microscopy, malaria RDTs as well as dried blood spots (DBSs) for molecular assay. Overall work flow of this study indicated in Fig. 1.

\section{Microscopy and malaria RDT}

The CareStart ${ }^{\mathrm{TM}}$ malaria RDTs (Pf/PV HRP2/PLDH) were used following manufacturer's instructions. Thick and thin blood smears were stained with a $10 \%$ buffer-diluted working solution of Giemsa for microscopic detection and the measurement of parasite density according to World Health Organization recommendations [24].

\section{Parasite DNA extraction and molecular analysis}

Genomic DNA was extracted from DBSs using the chelex-saponin method as described previously [25]. P. falciparum identification was confirmed by SYBR Green quantitative PCR (qPCR) assay after amplification of DNA coding for $18 \mathrm{~S}$ ribosomal RNA using species-specific primers [26]. After confirming P. falciparum positive

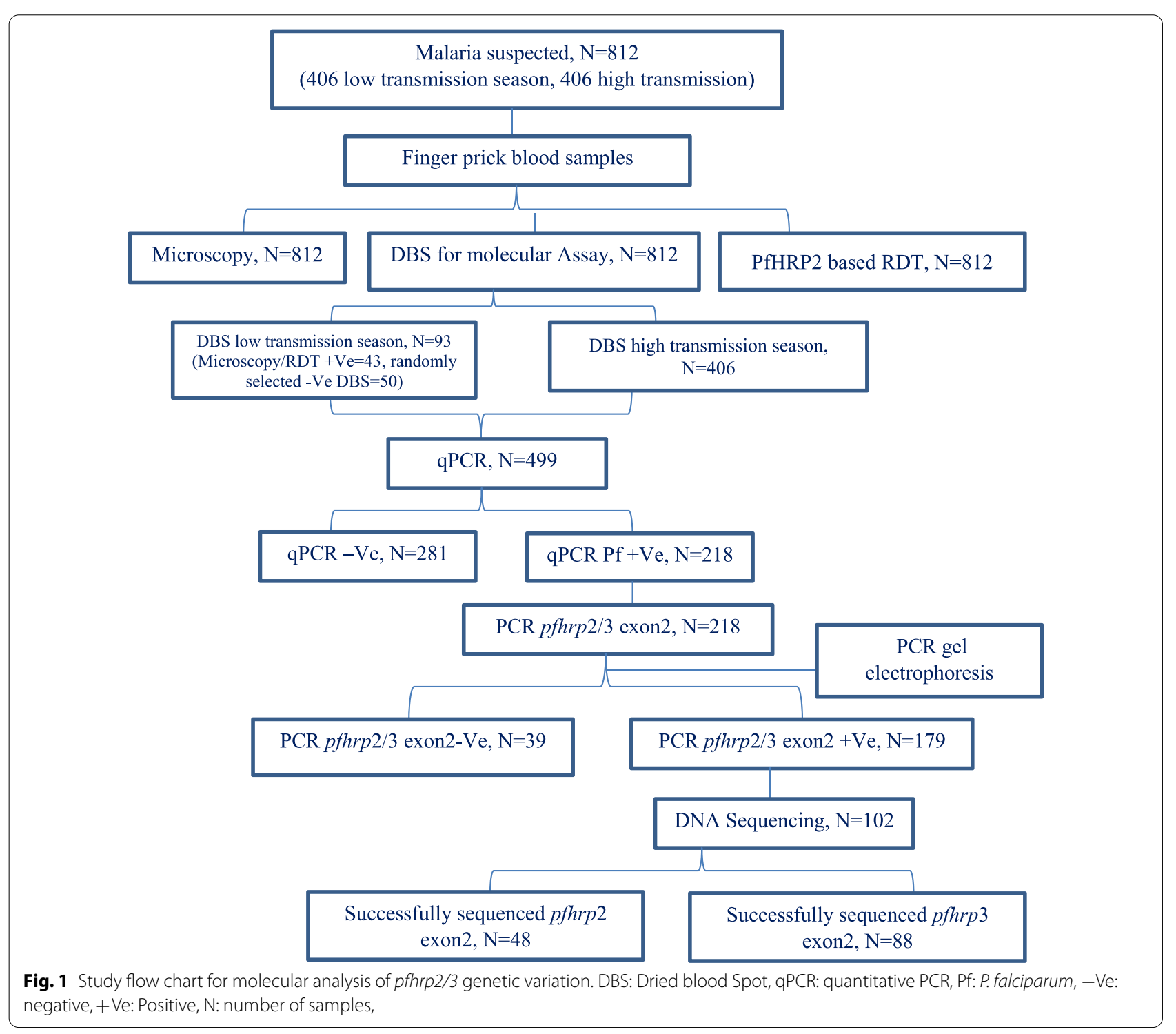


samples, all PCR reaction of pfhrp2 exon2 and pfhrp3 exon2 were performed at $25 \mu$ lotal with $2 \times$ Promega Hot Start Master Mix (Promega Corporation, Madison, USA), $0.4 \mu \mathrm{l}$ each of forward and reverse primers, and $3 \mu \mathrm{l}$ of extracted template DNA using PCR conditions previously described [17, 21, 27]. PCR products of $p f h r p 2$ exon 2 and pfhrp 3 exon 2 were separated by electrophoresis on a $2 \%$ agarose gel, visualized in a UV transilluminator, and the size of the expected amplicons compared to $1 \mathrm{~kb}$ DNA ladder. Primer sequences, PCR conditions, and expected amplicon size of pfhrp 2 exon 2 and $p f h r p 3$ exon 2 are provided in Additional file 2.

\section{Sequencing analyses}

A total of 48 pfhrp 2 exon 2 and 88 pfhrp 3 exon 2 high quality sequence data were included for molecular analysis of $p f h r p 2 / 3$ genetic variation. Pfhrp $2 / 3$ sequence of this study deposited in the NCBI Gen Bank database (with Accession number: MZ050658-MZ050792). All amplicons were cleaned using Exosap and sequenced using Sanger technology with ABI BigDyeTM Terminator v 3.1 chemistry (Thermofisher, Santa Clara, CA) and ran on a 3130 Genetic Analyzer (Thermofisher, Santa Clara, CA). Sequences were then cleaned and analysed using CodonCode Aligner Program V.6.0.2 (CodonCode Corporation, Centerville, MA). Nucleotide sequences were inputted into the ExPASy Translate Tool (Swiss Institute of Bioinformatics Resource Portal) and translated into corresponding amino acids sequence using the correct open reading frame. The amino acid repeat sequences in pfhrp 2 and $p f h r p 3$ were given a numeric code in the system as described previously $[8,11]$.

\section{Data analysis}

The proportions of each amino acid repeat of PfHRP2 and PfHRP3 in the P. falciparum isolates were analysed using Statistical Package for Social Sciences (SPSS) version 20. The association between PfHRP2 RDT results (sequenced samples could be RDT positive or negative) and certain group of amino acid repeat was tested by Chi square and Fisher's exact test. P-values $<0.05$ were interpreted as statistically significant. Bakers' model $[8$, 11] used for the prediction of PfHRP2 RDT sensitivity, the sequences of PfHRP2 were classified into four groups based on combined length of types $2 \times$ type 7 repeats. They include group $A(\geq 100$, very sensitive), group B (50-99, sensitive), group C ( $<43$, non-sensitive) and group I (44-49, borderline sensitive). The PfHRP2/3 amino acid sequences obtained from Ethiopia were compared with isolates from other countries based on the sequences deposited in the National Center for Biotechnology Information's (NCBI) GenBank using Basic Local Alignment Search Tool for Protein analysis (BLASTP).

\section{Results}

Genetic variation of PfHRP2 and PfHRP3 amino acids repeats

Among the 48 PfHRP2 sequenced samples, the length of pfhrp 2 exon 2 sequences varied between 453 and 873 base pairs (bp) in DNA and 150 to 290 residues in amino acids (aa). A total of 11 known PfHRP2 amino acid repeat types were identified (Table 1 ).

Nearly 65\% (31/48) of the distinct PfHRP2 amino acid sequence occurred only once, while five PfHRP2 amino acid sequence patterns $(\mathrm{I}-\mathrm{V})$ were found in more than one isolate (Fig. 2). The structural organizations of the amino acid repeats were found at different positions of the PfHRP2 amino acid sequences. PfHRP2 amino acid sequences of all the isolates started with a type 1 repeat. $89.6 \%$ of the PfHRP2 amino acid sequences ended with a type 10 repeat (occurred in five PfHRP2 patterns and distinct PfHRP2 sequence). 10.4\% of the PfHRP2 amino acid sequences contained a type 12 repeat (occurred only in four distinct PfHRP2 isolates). About 48\% (23/48) of the isolates had a PfHRP2 repeat motif composed of types 2 , $3,5,7,8,2$, and 7 , and this motif was absent in all PfHRP2 patterns. About 15\% (7/48) of the isolates had a PfHRP2 repeat motif composed of types $7,8,2$, and 7 , and this motif was found in all PfHRP2 patterns except pattern III (Fig. 2). Types 2 and 7 were the most frequent repeats among isolates and were broadly distributed in PfHRP2 amino acid sequence.

The type and frequency of each PfHRP2 amino acid repeat varied among parasite isolates at study sites. Each PfHRP2 amino acid sequence contains 15-36 repeats. Repeat types 1, 2 and 7 were found in $100 \%$ of the isolates, repeat types 6 and 10 were found in $95.8 \%$ of the isolates, and type 5, type 3 and type 8 were found in $75-91.7 \%$ of the isolates. Lower frequencies of PfHRP2 amino acid repeats were observed in type 4 (found in 20 isolates; $41.7 \%$ ), as well as types 12 and 13 (found in 5 isolates; 10.4\%). By contrast, types 9 and 11 were not identified in any PfHRP2 amino acid sequences in this study (Table 2).

Almost all PfHRP2 repeat types were found in all study sites with a slight difference in the frequency of repeat type within and between the study sites. Repeat types 1 , 2 and 7 were found in all isolates of Sherkole, Bambasi, Kurmuk, and Assosa. Repeat types 3, 5, 6, 8 and 10 were found in $66-100 \%$ isolates among study sites. Repeat types 4 and 12 occurred in $6.7-62.5 \%$ of the isolates among study sites. Type 13 was found only in Sherkole $(12.5 \% ; 3 / 24)$ and Bambasi $(13.3 \% ; 2 / 15)$ (Additional file 3$)$.

Among the 88 sequenced samples, the length of the pfhrp3 exon 2 ranged from 461 to 654 bp in DNA and 153 to 217 residues in amino acids. Of 88 PfHRP3 sequence 


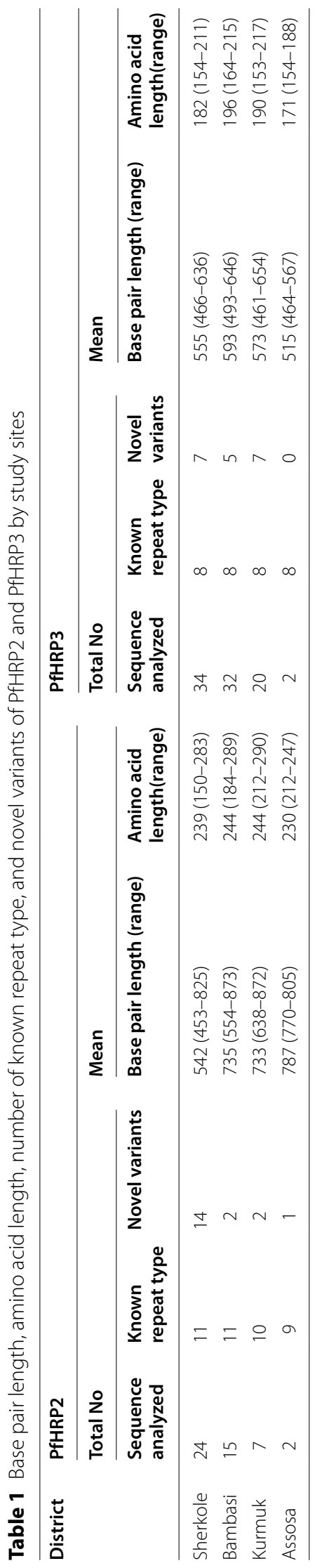




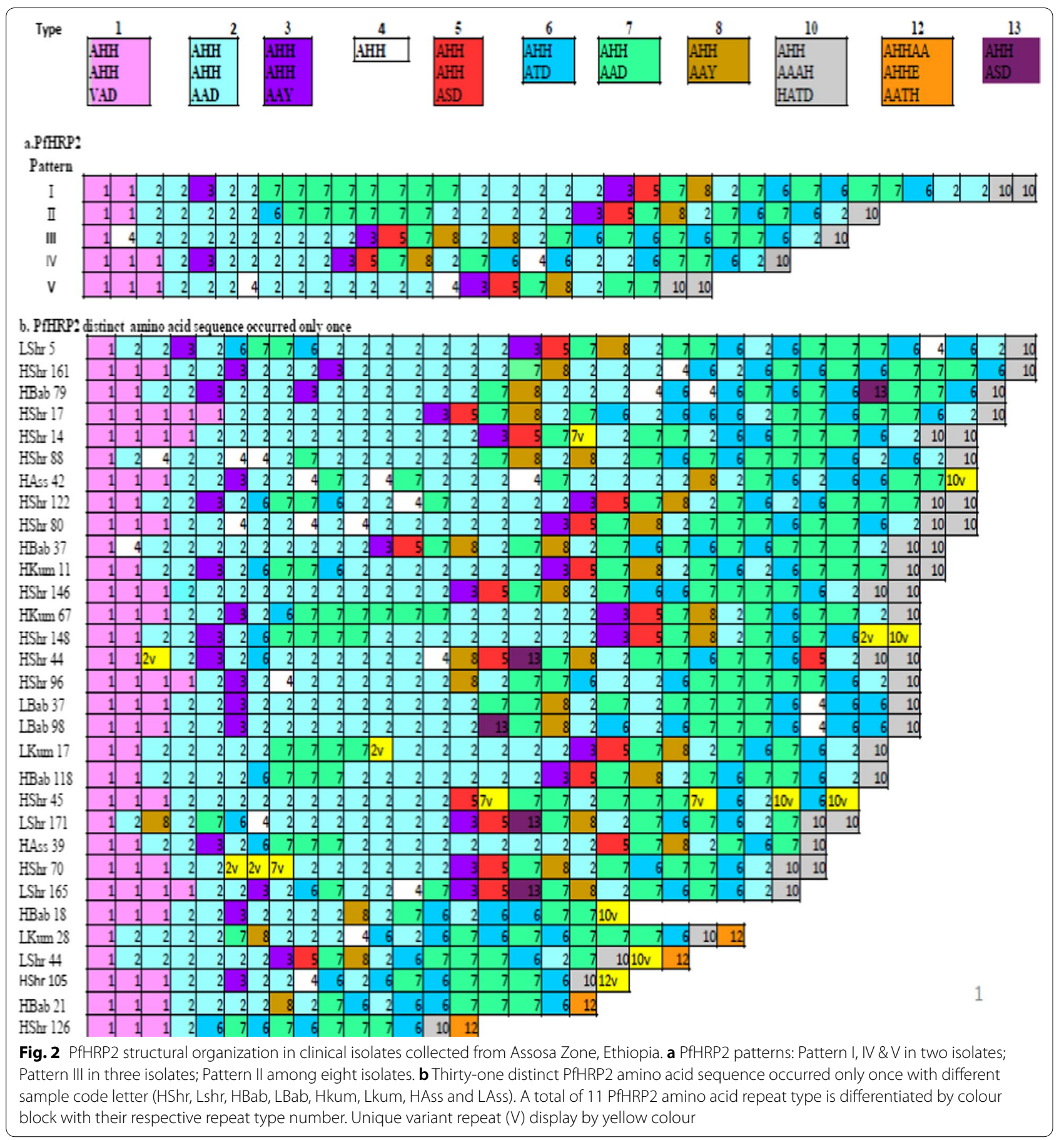

isolates, eight different PfHRP2 amino acid repeat types were identified (Table 1). Eight distinct PfHRP3 patterns detected in more than one isolate (Fig. 3). The PfHRP3 sequences began with type 1 (present in PfHRP3 pattern I-V), type 16 (present in PfHRP3 pattern VII-VIII), and type 15 (present in PfHRP3 pattern VI) repeats in 93.2\%, $4.6 \%$, and $2.3 \%$ of the isolates, respectively. The PfHRP3 sequences ended with type 4 (present in PfHRP3 pattern I-IV and VII-VIII), type 17 (present in PfHRP3 pattern $\mathrm{V}$ ), and type 18 (present in PfHRP3 pattern VI) repeats in $87.5 \%, 11.4 \%$, and $1.1 \%$ of the isolates, respectively.

All isolates of the PfHRP3 amino acid sequence in this study contained a non-repeating sequence (ANHGFHFNLHDNNSHTLHHAKANACFDD) between 
Table 2 Distribution of PfHRP2 and PfHRP3 Amino acids repeat in P. falciparum isolates in Assosa zone, Northwest Ethiopia

\begin{tabular}{|c|c|c|c|c|c|}
\hline \multirow[t]{2}{*}{ Type of repeat } & \multirow[t]{2}{*}{ Amino acid repeat sequence* } & \multicolumn{2}{|c|}{$\operatorname{PfHRP2}(n=48)$} & \multicolumn{2}{|c|}{ PfHRP3 $(n=88)$} \\
\hline & & No (\%) & Min to Max number & No (\%) & $\begin{array}{l}\text { Min to } \\
\text { Max } \\
\text { number }\end{array}$ \\
\hline 1 & AHHAHHVAD & $48(100)$ & $1-5$ & $83(94.3)$ & $0-3$ \\
\hline 2 & AHHAHHAAD & $48(100)$ & $1-15$ & $0(0.0)$ & 0 \\
\hline 3 & AHHAHHAAY & $42(87.5)$ & $0-2$ & $0(0.0)$ & 0 \\
\hline 4 & $\mathrm{AHH}$ & $20(41.7)$ & $0-3$ & 78 (88.6) & $0-1$ \\
\hline 5 & AHHAHHASD & $36(75.0)$ & $0-1$ & $0(0.0)$ & 0 \\
\hline 6 & AHHATD & $46(95.8)$ & $0-6$ & $0(0.0)$ & 0 \\
\hline 7 & AHHAAD & $48(100)$ & $3-13$ & 85 (96.6) & $0-2$ \\
\hline 8 & AHHAAY & $44(91.7)$ & $0-2$ & $0(0.0)$ & 0 \\
\hline 9 & AAY & $0(0.0)$ & $0-0$ & $0(0.0)$ & 0 \\
\hline 10 & AHHAAAHHATD & $46(95.8)$ & $0-2$ & $0(0.0)$ & 0 \\
\hline 11 & $\mathrm{AHN}$ & $0(0.0)$ & $0-0$ & $0(0.0)$ & 0 \\
\hline 12 & AHHAAAHHEAATH & $5(10.4)$ & $0-1$ & $0(0.0)$ & 0 \\
\hline 13 & AHHASD & $5(10.4)$ & $0-1$ & $0(0.0)$ & 0 \\
\hline 14 & AHHAHHATD & $0(0.0)$ & 0 & $0(0.0)$ & 0 \\
\hline 15 & AHHAHHAAN & $0(0.0)$ & $0-0$ & $84(95.5)$ & $0-2$ \\
\hline 16 & AHHAAN & $0(0.0)$ & $0-0$ & $88(100)$ & $7-16$ \\
\hline 17 & AHHDG & $0(0.0)$ & $0-0$ & $88(100)$ & $1-8$ \\
\hline 18 & AHHDD & $0(0.0)$ & $0-0$ & $87(98.9)$ & $0-4$ \\
\hline 19 & AHHAA & $0(0.0)$ & $0-0$ & $0(0.0)$ & 0 \\
\hline 20 & SHHDD & $0(0.0)$ & $0-0$ & 87 (98.9) & $0-2$ \\
\hline 21 & AHHAHHATY & $0(0.0)$ & $0-0$ & $0(0.0)$ & 0 \\
\hline 22 & AHHAHHAGD & $0(0.0)$ & $0-0$ & $0(0.0)$ & 0 \\
\hline 23 & ARHAAD & $0(0.0)$ & $0-0$ & $0(0.0)$ & 0 \\
\hline 24 & AHHTHHAAD & $0(0.0)$ & $0-0$ & $0(0.0)$ & $0-0$ \\
\hline
\end{tabular}

*PfHRP2 and PfHRP3 amino acid repeat motif as describe by Baker et al. [11]

$\mathrm{N}$ : Total number of PfHRP2 and PfHRP3 amino acid sequence in $P$. falciparum isolates

Min: Minimum number of amino acid repeat, Max: maximum number of amino acid repeat

types 7 and 20. Most of the isolates (96.6\%; 85/88) had a similar unique PfHRP3 repeat motif with structural organization of repeat types $15,16,20,17$, and 18 , and this motif was found in all PfHRP3 patterns except type VII (Fig. 3).

The total number of amino acid repeats and each repeat within the PfHRP3 sequence varied among the parasite isolates. Each PfHRP3 amino acid sequence consisted of 18-28 repeats. Repeats types 16 and 17 were found in $100 \%$ of the isolates. Types $1,4,7,15,18$, and 20 were detected in more than $88 \%$ of the isolates. In contrast, types $2-3,5-6,8-9,10-14$, and 19 were not identified in the PfHRP3 amino acid sequences in this study. All PfHRP3 repeat types were found in all study sites with some difference in the frequency of the repeat type within and between study sites. Repeat types 16, 17, 18, and 20 were found in $100 \%$ of the isolates in Sherkole, Bambasi, Kurmuk and Assosa. Types 18 and 20 in $95 \%$ of the isolates in Kurmuk. On the other hand, repeat types $1,4,7$, and 15 were found in $84.4-100 \%$ of the isolates among study sites (Additional file 3$)$.

\section{Analysis of Ethiopian PfHRP2/3 amino acid sequences against the global isolates}

BLAST analysis of PfHRP2/3 amino acid sequences were performed for each of the sequences obtained from Ethiopia to look for sequence similarities with other countries available from NCBI. PfHRP2 protein BLAST (BLASTP) analysis reveals a range of $88.50-98.82 \%$ similarity with $P$. falciparum isolates from Africa, India, and Myanmar, accession numbers from similar sequences are indicated in Additional file 4. Likewise, the BLASTP analysis of PfHRP3 reveals a range of $93.55-100 \%$ similarity with $P$. falciparum isolates from Kenya and India, accession numbers from similar sequences are indicated in Additional file 5. 


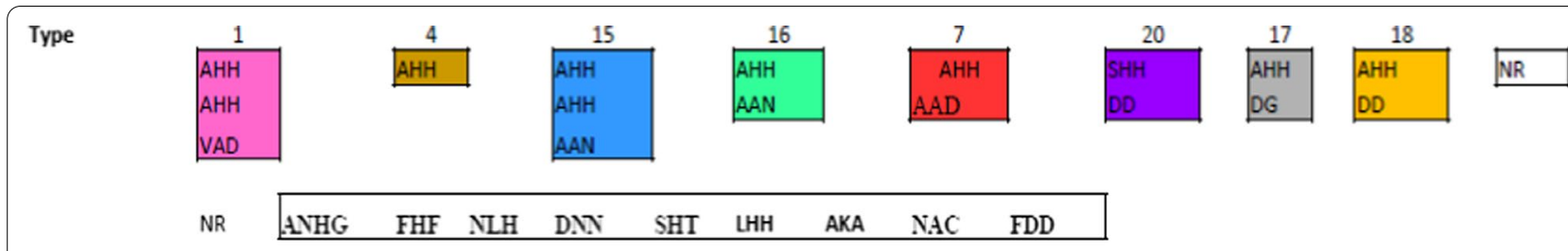

PFHRP3

Pattern

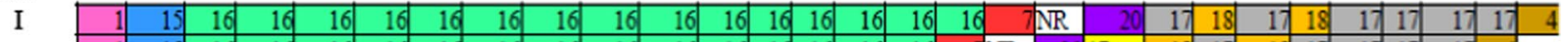

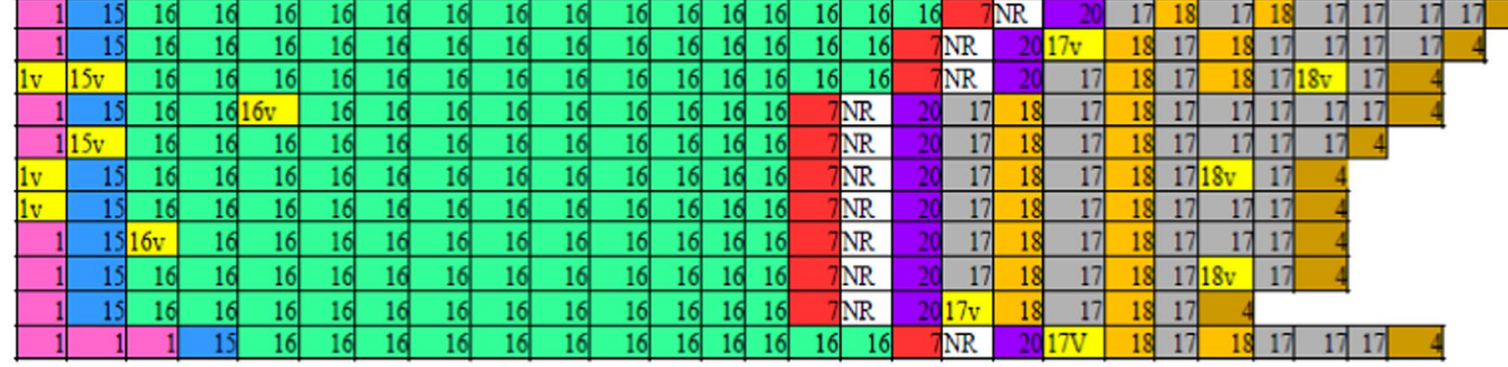

II \begin{tabular}{|l|l|l|l|l|l|l|l|l|l|l|l|l|l|l|l|l|l|l|l|}
\hline 1 & $15 \mathrm{v}$ & 16 & 16 & 16 & 16 & 16 & 16 & 16 & 16 & 16 & 16 & 16 & 16 & 16 & 16 & TNR & 20 & 17 & 4 \\
\hline
\end{tabular}

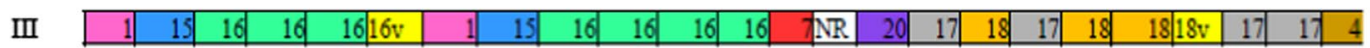

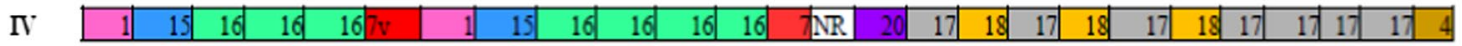

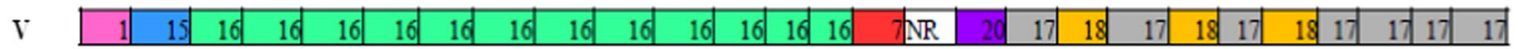

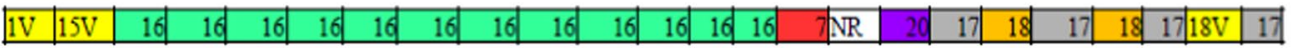

VI \begin{tabular}{|l|l|l|l|l|l|l|l|l|l|l|l|l|l|l|l|l|l|}
\hline 15 & 16 & 16 & 16 & 16 & 16 & 16 & 16 & 16 & 16 & 16 & 16 & $t_{N R}$ & 20 & 17 & 18 & 17 & 18 \\
\hline
\end{tabular}

VII \begin{tabular}{|l|l|l|l|l|l|l|l|l|l|l|l|l|l|l|l|l|l|l|l|l|}
\hline 16 & 16 & 16 & 16 & 16 & 16 & 16 & 16 & 16 & 16 & 16 & 16 & $7 \mathrm{NR}$ & 20 & 17 & 18 & 17 & 18 & 17 & 17 & 17 \\
\hline
\end{tabular}

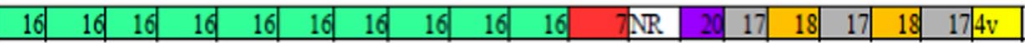

III \begin{tabular}{|l|l|l|l|l|l|l|l|l|l|l|l|l|l|l|l|l|l|l|l|l|}
\hline 16 & 16 & $16 / 7 \mathrm{v}$ & 17 & 15 & 16 & 16 & 16 & 16 & $7 \mathrm{NR}$ & 20 & 17 & 18 & 17 & 18 & 17 & 18 & 17 & 17 & 17 & 17 \\
\hline
\end{tabular}

Fig. 3 PfHRP3 structural organization in Assosa isolates. Eight PfHRP3 patterns identified in more than one isolates. A total of eight PfHRP3 amino acid repeat type is differentiated by color block with their respective repeat type number. Unique variant repeat (V) display by yellow color. NR: Non-repetitive region

\section{Comparison of novel variants of PfHRP2/3 repeat types in Ethiopia with global diversity}

A total of 18 novel variants of PfHRP2 repeat types were found in this study (Table 3). Of these novel PfHRP2 amino acid repeat sequences, 15 were not reported previously. Though type 7 (AHHDD) and type 10 (AHHAATHHATD, AHHAAAHHAND) repeats were reported previously (Additional file 6), they occurred at a very low frequency. Similarly, 13 new PfHRP3 repeat types were identified among the Ethiopian isolates. Among them, 12 were not previously reported. Only type 20 (SHHDG) was previously reported (Additional file 7) and it occurred at high frequency among the Ethiopian isolates.

\section{Effect of genetic variation of pfhrp2/3 on performance of RDTs}

Of the 48 P. falciparum samples included in Pfhrp2 exon2 sequence analysis, $72.9 \%(35 / 48)$ were detected as positive by PfHRP2 RDT. Among these PfHRP2 RDT positive samples, $82.9 \%(29 / 35)$ was found with a parasitaemia level $>250$ parasite $/ \mu \mathrm{L}$. However, all PfHRP2 RDT negative samples (13/48) were submicroscopic (Additional file 8). Similarly, among 88 P. falciparum samples included in Pfhrp3 exon2 sequence analysis, 79.5\% (70/88) was detected by PfHRP2 RDT and 84.3\% $(59 / 70)$ of these positive samples had parasitaemia levels $>250$ parasites $/ \mu$ l (Additional file 9). 
Table 3 List of Novel repeat /variants of PfHRP2 and PfHRP3 amino acid repeat types

\begin{tabular}{|c|c|c|c|c|c|}
\hline \multirow[t]{2}{*}{ Type of repeat } & \multirow[t]{2}{*}{ Known amino acid repeat } & \multicolumn{2}{|l|}{ PfHRP2 } & \multicolumn{2}{|l|}{ PfHRP3 } \\
\hline & & Novel repeat & Repeat frequency & Novel repeat & $\begin{array}{l}\text { Repeat } \\
\text { frequency }\end{array}$ \\
\hline \multirow[t]{3}{*}{1} & AHHAHHVAD & - & - & $\underline{\mathbf{D}} \mathrm{HHAHHVAD}$ & 1 \\
\hline & & & & $\underline{\mathbf{P}} \mathrm{HHAHHVAD}$ & 4 \\
\hline & & & & $\underline{\text { SPHAHHVAD }}$ & 1 \\
\hline \multirow[t]{6}{*}{2} & AHHAHHAAD & AHHAHHEAD & 1 & & \\
\hline & & $\mathrm{AHHA} \underline{\mathbf{Q}} \mathrm{HAAD}$ & 1 & & \\
\hline & & AHHAHHA $\underline{\mathbf{H}}$ & 1 & & \\
\hline & & APD & 1 & & \\
\hline & & AHHAH $\underline{\mathbf{V}} A A D$ & 1 & & \\
\hline & & AHHAHHAAY & 1 & & \\
\hline 3 & AHHAHHAAY & AHHAH $\underline{Y} A A Y$ & 1 & & \\
\hline 4 & $\mathrm{AHH}$ & & & $\underline{\mathbf{v}} \mathrm{HH}$ & 1 \\
\hline \multirow[t]{3}{*}{7} & AHHAAD & AHHA $\underline{\mathbf{V H}}$ & 1 & & \\
\hline & & AHHADDD & 2 & & \\
\hline & & $A H H A \underline{\mathbf{V}} D$ & 1 & & \\
\hline \multirow[t]{7}{*}{10} & AHHAAAHHATD & AHHAAAHHA ${ }^{\dagger}$ & 1 & & \\
\hline & & AHHAAA므HATD & 1 & & \\
\hline & & AHHAAAHQ $\underline{\mathbf{Q}} A T D$ & 1 & & \\
\hline & & AHHAAAHHAND & 1 & & \\
\hline & & AHHAAAHHA $\underline{\mathbf{R} D}$ & 1 & & \\
\hline & & AHHAA $\underline{\mathbf{D}} A H H A T D$ & 1 & & \\
\hline & & AHHAAI HHATD & 1 & & \\
\hline 12 & AHHAAAHHEAATH & $\underline{\mathbf{V}}$ HHAAAHHEA $\underline{\mathbf{P L C}}$ & 1 & & \\
\hline \multirow[t]{2}{*}{15} & AHHAHHAAN & - & & AHHAHHAㅁN & 3 \\
\hline & & - & & AHHAHH $\underline{\mathbf{V}} A N$ & 1 \\
\hline \multirow[t]{3}{*}{16} & AHHAAN & - & & $\mathrm{AHHA} \underline{\mathrm{GD}}$ & 4 \\
\hline & & - & & ALLAAN & 1 \\
\hline & & - & & $\underline{\mathbf{S}}$ HHAAN & 1 \\
\hline \multirow[t]{3}{*}{18} & AHHDD & & & $\underline{\mathbf{E}} \mathrm{HHDE}$ & 1 \\
\hline & & & & $\mathrm{AHHDE}$ & 3 \\
\hline & & & & $\mathrm{A} \underline{\mathbf{R}} \mathrm{HDD}$ & 1 \\
\hline 20 & SHHDD & & & SHHDG & 7 \\
\hline
\end{tabular}

The asterisks $(\dagger)$ show deletion position of amino acid code (double letter), bold underline letter indicate position of novel repeat with replacement of one or more amino acid compared to known repeat

The performance of PfHRP2 RDT and the number of PfHRP2 amino acid repeat $1(\mathrm{P}=0.944)$, repeat 2 $(\mathrm{P}=0.456)$, repeat $6(\mathrm{P}=0.95)$, repeat $7(\mathrm{P}=0.486)$, and repeat $10(\mathrm{P}=0.273)$ observed in the samples were not statistically associated with one another. Likewise, the performance of PfHRP2 RDT and variation of PfHRP3 amino acid repeat $1(\mathrm{P}=0.928)$, repeat $4(\mathrm{P}>0.05)$, repeat $7(\mathrm{P}>0.05)$, repeat $15(\mathrm{P}>0.05)$, repeat $18(\mathrm{P}=0.436)$, and repeat $20 \quad(\mathrm{P}>0.05)$ were not statistically associated. Interestingly, significant association was detected between performance of PfHRP2 and the number of PfHRP3 repeat $16(\mathrm{P}=0.031)$ and repeat $17(\mathrm{P}<0.001)$ (Additional file 10).
Amino acid changes in PfHRP2 and PfHRP3 repeat types observed in this study might affect the performance of PfHRP2 RDT. Eighteen novel PfHRP2 and 13 novel PfHRP3 repeat types were identified within 13 PfHRP2 and 24 PfHRP3 sequence in the P. falciparum population, respectively. Of 13 PfHRP2 sequences, $38.5 \%(5 / 13)$ that had the novel variants were detected negative by PfHRP2 RDT (Additional file 8). Of the 24 PfHRP3 sequences, $37.5 \%(9 / 24)$ that had the novel variants were negative by PfHRP2 RDT (Additional file 9). 
Prediction of PfHRP2 RDT sensitivity using Bakers' model According to the Baker's model, most of the isolates in this study was from group B $(54.2 \%, 26 / 48)$ followed by group A $(22.9 \%, 11 / 48)$. Among these isolates, $100 \%(8 / 8)$ in group A and 73.7\% (14/19) in group B were predicted to be detected by PfHRP2 RDT at parasitaemia level $>250$ parasite/ $\mu$ l. About $86 \%$ (6/7) of P. falciparum isolates in group $C$ were sensitive to PfHRP2 RDT at parasitaemia level $>250$ parasites $/ \mu$ in this study (Table 4 ).

\section{Discussion}

The present study provides valuable information on genetic variations in the PfHRP2 and PfHRP3 amino acid repeat types, which could affect the performance of PfHRP2 RDT for P. falciparum diagnosis. The structural organization of PfHRP2 amino acid sequences were less conserved than PfHRP3 among the Ethiopia isolates. The sequence length and the number of amino acid repeat types of PfHRP2 and PfHRP3 in this study were comparable to those previously reported in Yemen [28] and Madagascar [15], but lower than those in Kenya [29] and Ghana [30] and higher than in India [31].

All PfHRP2 repeat types started with repeat type 1 in the Ethiopian P. falciparum isolates, similar to previous reports in Kenya [29], Yemen [28], Senegal [32], and outside Africa [8]. The majorities of the PfHRP2 repeat ended with type 10 repeat which showed discordance with the previous reports from Africa [28, 29, 32] and Asia [31]. On the other hand, a small proportion of the isolates (10.4\%) ended with a type 12 PfHRP2 repeats coincide with reports from Senegal [33]. Two types of PfHRP2 repeat motifs structural organization repeat motifs $(2,3,5,7,8,2$, and 7$)$ and $(7,8,2,7)$, were identified $[8,29]$.

Eleven different PfHRP2 repeats and eight different PfHRP3 repeats were found at Sherkole, Bambasi, Kurmuk and Assosa with slight difference in the frequency of

Table 4 Frequency of PfHRP2 sequence groups based on the Baker's model and PfHRP2 RDT results $(n=48)$

\begin{tabular}{llll}
\hline Group & Frequency $(\mathbf{N}=48)$ & $\begin{array}{l}\text { Prediction of } \\
\text { PfHRP2 RDT } \\
\text { Positivity }\end{array}$ & $\begin{array}{l}\text { Microscopy } \\
\text { 250 parasite/ } \boldsymbol{\mu l}\end{array}$ \\
& No (\%) & No & No) \\
\hline A & $11(22.9)$ & $8(72.7)$ & $8(100)$ \\
B & $26(54.2)$ & $19(73.1)$ & $14(73.7)$ \\
C & $10(20.8)$ & $7(70.0)$ & $6(85.7)$ \\
I & $1(2.1)$ & $1(100)$ & $1(100)$
\end{tabular}

PfHRP2 sequence isolates were classified into four groups according to the frequency number of types $2 \times$ type 7 repeats: group $A(\geq 100$, very sensitive), group B (50-99, sensitive), group C ( $<43$, non-sensitive) and group I (44-49, borderline) as describe previously (Baker et al. [8]) the repeat type within and between $P$. falciparum isolates in Ethiopia, similar to those reported in Africa [29, 30] and worldwide [8].The PfHRP2 repeat types observed in the present study were previously reported in Africa [2830], Asia [8, 31], and America [34, 35]. BLAST analysis of the Ethiopia PfHRP2 and PfHRP3 amino acid sequences revealed the presence of similarities and shared identity with isolates from Kenya. Interestingly, the amino acid sequences similarities of PfHRP2 and PfHRP3 also extended beyond the border of Ethiopia, India, and Myanmar, which is also in agreement with a recent study from Ghana [30].

On the contrary, PfHRP2 repeat types obtained within this study showed certain difference from African and global reports in a number of ways. First, PfHRP2 type 12 repeat was found in a few isolates (10.5\%) this did not align with previous studies from Kenya [29], Ghana [30] and Central America [35]. However, studies from Senegal [33] showed the presence of type 12 repeats in a few isolates similar to the present study. Second, this study did not find type 9 and 11 repeats isolates which is consistent with most studies from African countries [28-30], but type 11 repeats were reported in a few isolates from Madagascar [15], while type 9 was reported from Senegalese isolates [33]. Third, types 14-27 were completely absent in all isolates in this study. These results disagree with other studies, which reported the rare occurrence of type 14 from Nigeria [8] and Madagascar [15], and type 19 from Kenya [29]. The possible explanation for such varied distribution repeat types may be due to random mutation and local selection or directional spread of deleted strains of $p f h r p 2 / 3$ throughout the world [36].

Concerning PfHRP3, the majorities of sequences started with type 1 and ended with a type 4 repeats in the Ethiopia isolates, which agrees with previous studies [29, 31]. Moreover, all isolates of PfHRP3 amino acid sequence contained a singleton non-repeating sequence [11]. The findings of PfHRP3 repeat types in the present study are consistent with previous reports from Kenya [29], Yemen [28], Ghana [30], and globally [8]. On the other hand, type 2 repeat was absent in all isolates of PfHRP3 sequence in Ethiopia as well as other parts of Africa [28-30], but type 2 repeat has been reported in PfHRP3 in few isolates from Kenya [29] and India [31]. This variation observed in PfHRP2 and PfHRP3 repeat types might be due to differences in geographical and transmission settings $[8,37,38]$, frequency of exposure of drug and level of immunity of study participants [39, 40], clinical versus asymptomatic study participants, and methods used for molecular analysis.

Interestingly, of all the novels repeat types identified in this study, 15 in PfHRP2 and 12 in PfHRP3 have not been reported elsewhere. Among all novel PfHRP2 
repeat types, two unique repeat types (type 7-AHHDD, type 10-AHHAATHHATD) and one unique repeat type (type-AHHAAAHHAND) were found with low frequency in Ethiopia and also in a previous study from Ghana [30] and Kenya [29]. Among all novel PfHRP3 repeat types, a unique repeat type (type 20- SHHDG) was detected with high frequency in Ethiopia as well as in Kenya [29], China-Myanmar border [41], and India [31]. The emergence of novel PfHRP2 and PfHRP3 repeat types at low frequency could be initiated by replacement or deletion of one or more amino acid repeat type [42-44].

Parasite densities and genetic variation $p f h r p 2 / 3$ gene are the two most important factors that affect the performance of PfHRP2 RDT $[8,16,21]$. In this study, a high proportion of PfHRP2 RDT positive samples was observed with parasitaemia $>250$ parasites/ $\mu$ l, but substantial numbers of PfHRP2 RDT false negative samples were detected in submicroscopic infections similar to previous reports $[8,45]$.

According to Baker's model, the Ethiopian isolates mostly belong to groups A and B were predicated to be detected by PfHRP2 RDT at parasitaemia level $>250$ parasite/ $\mu$ l and thus aligns with previous studies found in Senegal [33], Madagascar [15], and India [46]. Interestingly, most of $P$. falciparum isolates in group $C$ were detectable by PfHRP2 RDT at parasitaemia level $\geq 200$ parasite $/ \mu$ l. This finding partially disagrees with the Baker' model [11] that predicts if the length of repeat types 2 and 7 is below 43 (as in group C), it will alter detection sensitivity of PfHRP2 RDT and lead to false negative results [33].

In this study, the length of amino acid repeat in PfHRP2 (type 1, 2, 7) and shared repeats in PfHRP2 and PfHRP3 (type $1,4,7$ ) were not statistically associated with the performance of PfHRP2 RDT, consistent with previous study [29]. Instead, PfHRP2 RDT positivity was significantly improved as the length of PfHRP3 repeat type 16 and 17 increased. Types 1,4 and 7 were common repeat types in both PfHRP2 and PfHRP3 amino acid sequence, whereas types 16 and 17 were identified only in PfHRP3 in high frequency among all isolates. In line with this, PfHRP2 and PfHRP3 exhibit structural homology as exon 2 in both $p f h r p 2$ and pfhrp3 encodes similar amino acids and cross reaction may play a role in the diagnosis of falciparum malaria [7, 12]. Moreover, novel PfHRP2 and PfHRP3 repeat variants detected in this study might influence the binding affinity to monoclonal antibody and affect the sensitivity of PfHRP2 RDTs [12]. As results, considerable proportions of false negative results were found in this study by PfHRP2 RDT, 38.5\% of PfHRP2 and 37.5\% of PfHRP3 sequence with novel variants.

\section{Limitations}

This study had two limitations. First, the samples represented a limited geographical area from Assoa, Ethiopia. Second, this study did not assess cross reactivity of the possible epitopes based on the amino acid repeat sequence of PfHRP2 and PfHRP3 using specific monoclonal antibodies.

\section{Conclusion}

The present study indicated, for the first time, the presence of extensive existence of genetic variability of PfHRP2 and PfHRP3 amino acid repeats including novel repeats in P. falciparum isolates within and between the study sites in Ethiopia. There is a need to closely monitor the performance of PfHRP2 RDT and examine the distribution of novel repeat type and shared repeat in PfHRP2/3, and unique repeat found in PfHRP3 broadly in Ethiopia.

\section{Abbreviations}

RDT: Rapid diagnostic tests RDT; HRP2: Histidine-Rich Protein 2/3; pfhrp2/3gene: P. falciparum Histidine-rich protein 2 and 3 genes; DBS: Dried blood spots; PCR: Polymerase chain reaction; qPCR: Quantitative polymerase chain reaction; SPSS: Statistical Package for Social Sciences; NCBI: National Center for Biotechnology Information's; BLASTP: Basic Local Alignment Search Tool for Protein analysis.

\section{Supplementary Information}

The online version contains supplementary material available at https://doi. org/10.1186/s12936-021-03928-3.

Additional file 1. The map showing the study area in Assosa zone. The map generated using ArcGIS version 10.0 software.

Additional file 2. Primer sequences, PCR conditions and expected amplicon sizes of Pfhrp2 exon2 and Pfhrp3 exon2.

Additional file 3. Distribution of PfHRP2 and PfHRP3 amino acid repeat by study site.

Additional file 4. BLASTP of Ethiopian PfHRP2 sequences for 4 Pattern and 25 distinct haplotype isolates.

Additional file 5. BLASTP of Ethiopian PfHRP3 sequences for eight different Pattern.

Additional file 6. Comparison of Ethiopia PfHRP2 novel repeat with other countries.

Additional file 7. Comparisons of Ethiopian PfHRP3 novel variants with others.

Additional file 8. Baker repeats types and P. falciparum infection diagnosis. PfHRP2 sequence isolates classified into four groups according to the frequency number of types $2 \times$ type 7 repeats: group $A$ ( $\geq 100$, very sensitive), group $B(50-99$, sensitive), group $C(<43$, non-sensitive) and group I (44-49, borderline) as describe previously (Baker et al, 2010).

Additional file 9. PfHRP2 RDT and microscopy results in Pfhrp3 sequence isolates.

Additional file 10. Effect of the number of PfHRP2 and PfHRP3 amino acid repeat on the performance of PfHRP2 RDT. 


\section{Acknowledgements}

The authors sincerely thank Addis Ababa University, Aklilu Lemma Institute of Pathbiology, University of North Carolina at Charlotte (Department of Bioinformatics and Genomics, Department of Biological Sciences). Study participants are also duly acknowledged for their voluntary participation. The authors would also appreciate the field data collectors and laboratory experts who participated during the study.

\section{Authors' contributions}

GA, AL, EL, DJ and LG conceived and designed the study. GA collected the sample. GA, KB and KL performed laboratory analysis under the direction of LG, DJ and EL. All authors read and approved the final manuscript.

\section{Funding}

This study was supported in part by Addis Ababa University for data collection, University of North Carolina at Charlotte (Department of Bioinformatics and Genomics, College of Computing and Informatics, Bioinformatics Research Center, Department of Biological Sciences) for molecular laboratory work. The content is the sole responsibility of the authors. The funder had no role in the study design, data collection and analysis, the decision to publish, or the preparation of the manuscript.

\section{Availability of data and materials}

The datasets used and/or analysed during in this study are included in this article and available from the corresponding author on reasonable request.

\section{Declarations}

\section{Ethics approval and consent to participate}

Ethical clearance was obtained from the institutional review board of the Aklilu Lemma Institute of Pathobiology, Addis Ababa University (Ref No ALIPB/ IRB006/2017/18) and also from the Institutional Review Board of the University of North Carolina at Charlotte (IRB number 18-0451). Material transfer agreements were made between Addis Ababa University and the University of North Carolina at Charlotte. Blood samples were taken from finger-prick after obtaining written informed consent and/or assent from parents or guardian for children. Health workers treated positive malaria cases according to national treatment guidelines.

\section{Consent for publication}

This study reports no individual person's data. There is no opposition to its presentation and/or publication. All authors have read and approved the manuscript for publication.

\section{Competing interests}

The authors declare that they have no conflict of interests.

\section{Author details}

${ }^{1}$ Addis Ababa University, Aklilu Lemma Institute of Pathobiology, Addis Ababa, Ethiopia. ${ }^{2}$ Departments of Bioinformatics and Genomics, University of North Carolina at Charlotte, Charlotte, NC 28223, USA. ${ }^{3}$ Department of Biological Sciences, University of North Carolina at Charlotte, Charlotte, NC 28223, USA. ${ }^{4}$ School of Data Sciences, University of North Carolina at Charlotte, Charlotte, NC 28223, USA.

Received: 3 June 2021 Accepted: 26 September 2021

Published online: 09 October 2021

\section{References}

1. WHO. World malaria report 2019. Geneva: World Health Organization; 2019.

2. Cowman AF, Healer J, Marapana D, Marsh K. Malaria: biology and disease. Cell. 2016;167:610-24.

3. WHO. Global Technical Strategy for Malaria 2016-2030. Geneva: World Health Organization; 2015.

4. WHO. Good practices for selecting and procuring rapid diagnostic tests for malaria. Geneva: World Health Organization; 2011.
5. Murray CK, Gasser RA, Magill AJ, Miller RS. Update on rapid diagnostic testing for malaria. J Clin Microbiol. 2008;21:97-110.

6. Jain P, Chakma B, Patra S, Goswami P. Potential biomarkers and their applications for rapid and reliable detection of malaria. Biomed Res Int. 2014:2014:852645.

7. Wellems TE, Howard RJ. Homologous genes encode two distinct histidine-rich proteins in a cloned isolate of Plasmodium falciparum. Proc Natl Acad Sci USA. 1986;83:6065-9.

8. Baker J, Ho M-F, Pelecanos A, Gatton M, Chen N, Abdullah S, et al. Global sequence variation in the histidine-rich proteins 2 and 3 of Plasmodium falciparum: implications for the performance of malaria rapid diagnostic tests. Malar J. 2010;9:129.

9. Sharma Y. Genomic organization, structure and possible function of histidine-rich proteins of malaria parasites. Int J Biochem. 1988;20:471-7.

10. Rock E, Marsh K, Saul A, Wellems T, Taylor DW, Maloy W, et al. Comparative analysis of the Plasmodium falciparum histidine-rich proteins HRP-I, HRP-II and HRP-III in malaria parasites of diverse origin. Parasitology. 1987:95:209-27.

11. Baker J, McCarthy J, Gatton M, Kyle DE, Belizario V, Luchavez J, et al. Genetic diversity of Plasmodium falciparum histidine-rich protein 2 (PfHRP2) and its effect on the performance of PfHRP2-based rapid diagnostic tests. J Infect Dis. 2005;192:870-7.

12. Lee N, Baker J, Andrews KT, Gatton ML, Bell D, Cheng Q, et al. Effect of sequence variation in Plasmodium falciparum histidine-rich protein 2 on binding of specific monoclonal antibodies: Implications for rapid diagnostic tests for malaria. J Clin Microbiol. 2006;44:2773-8.

13. Levinson G, Gutman G. Slipped-strand mispairing: a major mechanism for DNA sequence evolution. Mol Biol Evol. 1987;4:203-21.

14. Baker J, Gatton ML, Peters J, Ho M-F, McCarthy JS. Transcription and expression of Plasmodium falciparum histidine-rich proteins in different stages and strains: implications for rapid diagnostic tests. PLoS ONE. 2011;6:e22593

15. Mariette N, Barnadas C, Bouchier C, Tichit M, Ménard D. Country-wide assessment of the genetic polymorphism in Plasmodium falciparum and Plasmodium vivax antigens detected with rapid diagnostic tests for malaria. Malar J. 2008;7:219.

16. Cheng Q, Gatton ML, Barnwell J, Chiodini P, McCarthy J, Bell D, et al. Plasmodium falciparum parasites lacking histidine-rich protein 2 and 3: a review and recommendations for accurate reporting. Malar J. 2014;13:283.

17. Alemayehu GS, Blackburn K, Lopez K, Dieng CC, Lo E, Janies D, et al. Detection of high prevalence of Plasmodium falciparum histidine-rich protein 2/3 gene deletions in Assosa zone, Ethiopia: implication for malaria diagnosis. Malar J. 2021;20:109.

18. Beshir KB, Sepúlveda N, Bharmal J, Robinson A, Mwanguzi J, Busula $A O$, et al. Plasmodium falciparum parasites with histidine-rich protein 2 (pfhrp2) and pfhrp3 gene deletions in two endemic regions of Kenya. Sci Rep. 2017;7:14718.

19. Hamid MA, Awad-Elgeid M, Nasr A. Gene variation and suspected Plasmodium falciparum histidine-rich protein 2 gene deletion and its impact on sensitivity of malaria rapid diagnostic tests in Sudan. BMJ Glob Health. 2017;2:A21.

20. Amoah $L E$, Abankwa J, Oppong A. Plasmodium falciparum histidine rich protein-2 diversity and the implications for PfHRP 2: based malaria rapid diagnostic tests in Ghana. Malar J. 2016;15:101.

21. WHO. Protocol for estimating the prevalence of pfhrp2/pfhrp3 gene deletions among symptomatic falciparum patients with false-negative RDT results. Geneva: World Health Organization; 2018.

22. Daniel WW, Cross CL. Biostatistics: a foundation for analysis in the health sciences. New York: John Wiley and Sons; 2018

23. Geleta G, Ketema T. Severe malaria associated with Plasmodium falciparum and P. vivax among children in Pawe Hospital, Northwest Ethiopia. Malar Res Treat. 2016;2016:1240962.

24. WHO. Malaria microscopy quality assurance manual-version 2. Geneva: World Health Organization; 2016.

25. Baidjoe A, Stone W, Ploemen I, Shagari S, Grignard L, Osoti V, et al. Combined DNA extraction and antibody elution from filter papers for the assessment of malaria transmission intensity in epidemiological studies. Malar J. 2013;12:272.

26. Rougemont M, Van Saanen M, Sahli R, Hinrikson HP, Bille J, Jaton K. Detection of four Plasmodium species in blood from humans by $18 \mathrm{~S}$ rRNA gene 
subunit-based and species-specific real-time PCR assays. J Clin Microbiol. 2004;42:5636-43.

27. Parr JB, Anderson O, Juliano JJ, Meshnick SR. Streamlined, PCR-based testing for pfhrp2-and pfhrp3-negative Plasmodium falciparum. Malar J. 2018;17:137.

28. Atroosh WM, Al-Mekhlafi HM, Al-Jasari A, Sady H, Al-Delaimy AK, Nasr NA, et al. Genetic variation of pfhrp2 in Plasmodium falciparum isolates from Yemen and the performance of HRP2-based malaria rapid diagnostic test. Parasit Vectors. 2015:8:388.

29. Nderu D, Kimani F, Thiong'o K, Karanja E, Akinyi M, Too E, et al. Plasmodium falciparum histidine-rich protein (PfHRP2 and 3) diversity in Western and Coastal Kenya. Sci Rep. 2019;9:1709.

30. Addai-Mensah O, Dinko B, Noagbe M, Ameke SL, Annani-Akollor ME, Owiredu E-W, et al. Plasmodium falciparum histidine-rich protein 2 diversity in Ghana. Malar J. 2020;19:256.

31. Bharti PK, Singh Chandel H, Krishna S, Nema S, Ahmad A, et al. Sequence variation in Plasmodium falciparum histidine rich proteins 2 and 3 in Indian isolates: implications for malaria rapid diagnostic test performance. Sci Rep. 2017;7:1308.

32. Deme AB, Park DJ, Bei AK, Sarr O, Badiane AS, Gueye PE, et al. Analysis of pfhrp2 genetic diversity in Senegal and implications for use of rapid diagnostic tests. Malar J. 2014;13:34.

33. Wurtz N, Fall B, Bui K, Pascual A, Fall M, Camara C, et al. Pfhrp2 and pfhrp3 polymorphisms in Plasmodium falciparum isolates from Dakar, Senegal: impact on rapid malaria diagnostic tests. Malar J. 2013;12:34.

34. Abdallah JF, Okoth SA, Fontecha GA, Torres REM, Banegas El, Matute ML, et al. Prevalence of pfhrp2 and pfhrp3 gene deletions in Puerto Lempira. Honduras Malar J. 2015;14:19.

35. Fontecha G, Pinto A, Escobar D, Matamoros G, Ortiz B. Genetic variability of Plasmodium falciparum histidine-rich proteins 2 and 3 in Central America. Malar J. 2019:18:31.

36. Baker J, Gatton ML, Peters J, Ho M-F, McCarthy JS, Cheng Q. Transcription and expression of Plasmodium falciparum histidine-rich proteins in different stages and strains: implications for rapid diagnostic tests. PLOS ONE. 2011:6:e22593.

37. Watson OJ, Verity R, Ghani AC, Garske T, Cunningham J, Tshefu A, et al. Impact of seasonal variations in Plasmodium falciparum malaria transmission on the surveillance of pfhrp2 gene deletions. Elife. 2019;8:e40339.
38. Pringle JC, Wesolowski A, Berube S, Kobayashi T, Gebhardt ME, Mulenga $M$, et al. High Plasmodium falciparum genetic diversity and temporal stability despite control efforts in high transmission settings along the international border between Zambia and the Democratic Republic of the Congo. Malar J. 2019;18:400.

39. Markwalter CF, Mudenda L, Leelawong M, Kimmel DW, Nourani A, Mbambara S, et al. Evidence for histidine-rich protein 2 immune complex formation in symptomatic patients in Southern Zambia. Malar J. 2018;17:256.

40. Apinjoh TO, Ouattara A, Titanji VP, Djimde A, Amambua-Ngwa A. Genetic diversity and drug resistance surveillance of Plasmodium falciparum for malaria elimination: is there an ideal tool for resource-limited sub-Saharan Africa? Malar J. 2019;18:217.

41. Li P, Xing H, Zhao Z, Yang Z, Cao Y, Li W, et al. Genetic diversity of Plasmodium falciparum histidine-rich protein 2 in the China-Myanmar border area. Acta Trop. 2015;152:26-31.

42. Akinyi S, Hayden T, Gamboa D, Torres K, Bendezu J, Abdallah JF, et al. Multiple genetic origins of histidine-rich protein 2 gene deletion in Plasmodium falciparum parasites from Peru. Sci Rep. 2013;3:2797.

43. Kemp D, Thompson J, Walliker D, Corcoran L. Molecular karyotype of Plasmodium falciparum: conserved linkage groups and expendable histidine-rich protein genes. Proc Natl Acad Sci USA. 1987;84:7672-6.

44. Gamboa D, Ho M-F, Bendezu J, Torres K, Chiodini PL, Barnwell JW, et al. A large proportion of $P$. falciparum isolates in the Amazon region of Peru lack pfhrp2 and pfhrp3: implications for malaria rapid diagnostic tests. PLOS ONE. 2010;5:e8091.

45. WHO. Malaria rapid diagnostic test performance: results of WHO product testing of malaria RDTs: round 7 (2015-2016). Geneva: World Health Organization; 2017.

46. Kumar N, Singh JP, Pande V, Mishra N, Srivastava B, Kapoor R, et al. Genetic variation in histidine rich proteins among Indian Plasmodium falciparum population: possible cause of variable sensitivity of malaria rapid diagnostic tests. Malar J. 2012;11:298.

\section{Publisher's Note}

Springer Nature remains neutral with regard to jurisdictional claims in published maps and institutional affiliations.
Ready to submit your research? Choose BMC and benefit from:

- fast, convenient online submission

- thorough peer review by experienced researchers in your field

- rapid publication on acceptance

- support for research data, including large and complex data types

- gold Open Access which fosters wider collaboration and increased citations

- maximum visibility for your research: over $100 \mathrm{M}$ website views per year

At BMC, research is always in progress.

Learn more biomedcentral.com/submissions 